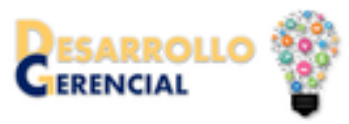

\title{
Intervención en el clima laboral para el desarrollo organizacional de una empresa de servicios logísticos
}

\author{
Action plan related to labor climate useful for the organizational \\ development of the logistics services company
}

\author{
Sofía Meza De los Cobos \\ Josefina Morgan Beltrán \\ Elía Socorro Díaz Nieto iD \\ Universidad Autónoma de Querétaro, Querétaro, México.
}

\begin{abstract}
Resumen
Objetivo: Realizar un diagnóstico de clima organizacional para sugerir un plan de intervención para la organización. Método: El método utilizado fue el estudio de caso y la fenomenología por medio de entrevistas, la observación y análisis de documentos. Resultados y Discusiones: Los datos obtenidos pretenden descubrir la ventaja competitiva organizacional, pero sobretodo las áreas de oportunidad tanto en lo administrativo y tecnoestructural, como en los procesos humanos. Los resultados arrojan que el desarrollo organizacional se valora como una herramienta administrativa para incrementar productividad, reducir riesgos y ambigüedades en los procesos como también para generar modificaciones en la organización mediante diagnósticos de mejora continua. Conclusiones: el clima laboral repercute directamente en la eficiencia y eficacia de las organizaciones y en el desempeño de su personal.

Palabras clave: Clima laboral, Desarrollo organizacional, Empresa de servicios logísticos.

Clasificación JEL: M12, M5, M54
\end{abstract}

Autores de Correspondencia

sofiamc9@gmail.com jmorganbeltran@yahoo.com.mx eliadiaznieto@gmail.com

Recibido: 24-04-18

Aceptado: $30-10-18$

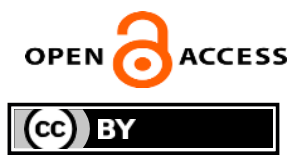

\section{Abstract}

Objective: this paper aims to make a diagnosis related to the organizational labor climate in order to suggest an action plan useful for the organization. Method: the case study, phenomenology through interviews, observation and analysis of documents, were used as method. Results and discussions: The data collected intends to discover not only the organizational competitive advantage, but also all those opportunity areas; both administratively and technologically, as well as in human processes. The results show that organizational development is considered as an administrative tool to increase productivity, reduce risks and ambiguities in processes as well as to generate changes in the organization through continuous improvement diagnostics. Conclusions: the labor climate has a direct impact on organizations' efficiency and effectiveness and on their staff's job performance.

Keywords: Labor climate, Organizational development, Logistic Services Company JEL Classification: M12, M5, M54

Copyright (C) 2019

Desarrollo Gerencial

Como citar este artículo (Apa):

Meza, S., Morgan, J. y Díaz, E. (2019). Intervención en el clima laboral para el desarrollo organizacional de una empresa de servicios logísticos. Desarrollo Gerencial, 11(1), 9-32. DOI: https://doi.org/10.17081/dege.11.1.3010 


\section{Introducción}

El diagnóstico organizacional constituye una parte de gran importancia en el proceso de consultoría y a partir de un diagnóstico acertado se podrán implementar cambios deseados dentro de una organización basados en tendencias de desarrollo, proyecciones y planificaciones que ayuden al desarrollo y bienestar de la misma.

En el presente estudio se ha expuesto una visión panorámica de los principales temas del diagnóstico organizacional, así como la implementación de este mismo en una empresa de servicios logísticos ubicada en la ciudad de Querétaro, estado de Querétaro, México, para así determinar las intervenciones tanto administrativas, tecno estructurales como en procesos humanos necesarias en el clima laboral actualmente vivido en la organización para así encaminar y cumplir los objetivos de la organización y las necesidades de la misma.

La investigación se centra en el desarrollo de un plan de diagnóstico en la empresa Servicios Logísticos H S.A. de C.V. en la oficina de Querétaro acorde a las técnicas de desarrollo organizacional.

Servicios Logísticos H S.A. de C.V. Querétaro se encuentra en un constante crecimiento dado a que de 2015 a 2016 se abrieron 9 vacantes nuevas en los diferentes departamentos como a nivel Gerencial, servicio al cliente, equipo de operaciones y ventas debido a la alta demanda que los servicios logísticos están siendo requeridos a nivel mundial y por lo mismo la empresa debe asegurar que la calidad y el servicio brindado a los clientes sea el adecuado con el objetivo de continuar con esta tendencia de crecimiento y lealtad de sus clientes.

El clima organizacional afecta a procesos organizacionales y psicológicos influyendo en la productividad, satisfacción laboral y el bienestar de los trabajadores; Bordas (2016) comenta que el capital humano en un buen contexto laboral es la principal fuente de ventajas competitivas sostenibles en las empresas y organizaciones. Por estos motivos Servicios Logísticos H S.A. de C.V. se encuentra en la necesidad de encontrar situaciones que estén afectando en la organización con el fin de proponer soluciones o modelos que orienten la implementación de estrategias para fortalecer el desarrollo humano, mejorar la percepción y ambiente organizacional debido a que cada uno de los miembros de la organización tiene una participación clave en el crecimiento y desarrollo de la compañía por lo que ha decidido aplicar el proceso de diagnóstico a cada uno de los departamentos de la oficina de Querétaro. 


\section{Fundamentación teórica}

\section{Desarrollo Organizacional.}

Guízar (2013) en su investigación sobre desarrollo organizacional menciona a Beckhard (1996) como un autor destacado en el tema y el cual menciona que el desarrollo organizacional es un esfuerzo planificado que involucra a toda la organización y es controlado desde el nivel más alto con la finalidad de incrementar la efectividad y sobretodo el bienestar de la organización mediante intervenciones planificadas y aplicando los conocimientos de las ciencias de la conducta. "El desarrollo organizacional es una mezcla de ciencia y arte, lo cual lo convierte en una disciplina apasionante" (Guízar, 2013, p.6).

Otras definiciones destacadas sobre Desarrollo Organizacional que menciona Guízar $(2013$, p.6) son las siguientes:

Porras y Robertson (1992) afirma que:

El desarrollo organizacional es una serie de teorías, valores, estrategias y técnicas basadas en las ciencias de la conducta y orientada al cambio planificado del escenario de trabajo de una organización, con el propósito de incrementar el desarrollo individual y de mejorar el desempeño de la organización mediante la alteración de las conductas de los miembros de la organización en el trabajo.

Burke (1994) sostiene que es un proceso de cambio planificado en la cultura de una organización, mediante la utilización de tecnologías, las ciencias de la conducta, la investigación y la teoría.

Guízar (2013) describe los elementos que definen al desarrollo organizacional siendo estos:

- Intervenciones. Herramientas o medios de los que se vale el desarrollo organizacional para llevar a cabo el cambio planeado.

- Consultor. También llamado facilitador o agente de cambio (externo o interno) el cual es responsable, junto con la alta dirección, de llevar a cabo el programa de desarrollo organizacional estimulando y coordinando el proceso.

- Sistema. Conjunto de elementos interrelacionados y que actúan de manera ordenada y armónica.

- Sistema-cliente. Organización donde se lleva a cabo el proceso de desarrollo organizacional. 
- Catarsis. Reacción que provoca el cambio que se lleva a cabo en la organización, es decir, es la reacción para cambiar antes ciertas circunstancias que obligan a esta misma a realizarlo.

- Conflicto proactivo. Tiene la finalidad de obtener resultados positivos para la organización, es decir, proporcionar un enfoque funcional a la organización y sete mismo puede ser provocado por el consultor.

- Cambio. Implica redefinir creencias, actitudes, valores, estrategias y prácticas con el objetivo de que la organización pueda adaptarse mejor a los cambios imperantes en el medio.

- Teoría del caos. Cada departamento puede llevar a cabo cambios o enfrentar situaciones no previstas, pero siempre estas deben de estar regidas por la misión, visión y políticas de la empresa.

- Transformación organizacional. Es una extensión del desarrollo organizacional, Administración del cambio; se centra en el valor de los costos, la calidad y los programas. Caracteriza al DO por la transferencia de conocimientos y habilidades para que al sistema le sea más fácil manejar el cambio en el futuro.

\section{Objetivos y valores del desarrollo organizacional.}

De Faria (2004) puntualiza diversos objetivos que todo desarrollo organizacional debe llegar a alcanzar siendo los más relevantes. (p. 44)

- Crear un clima de receptividad para reconocer las realidades organizacionales para diagnosticar y solucionar problemas.

- Establecer un clima de confianza y respeto entre jefes, colegas y subordinados.

- Desarrollar las potencialidades de los individuos y la capacidad de colaboración de los miembros de la organización.

- Compatibilizar, visualizar, armonizar e integrar las necesidades y objetivos de la empresa y de quienes forman parte de ella.

- Estimular las emociones y sentimientos de las personas debido que estos son benéficos o perjudiciales para la eficiencia y salud de la organización.

- Colocar los conflictos y tensiones ante los miembros en un ambiente controlado para que exista una retroalimentación racional y constructiva.

- Examinar el cómo, cuándo, dónde y cuánto las concepciones, el clima y cultura influyen sobre los objetivos, métodos, procesos, comportamientos, desempeños y resultados obtenidos

- Perfeccionar el sistema. 
Hernández, Gallarzo, Espinoza (2011) mencionan que los valores establecen las bases para la comprensión de las actitudes y motivación y además de que influyen en las percepciones de las personas.

Precisamente por tal motivo, Ulibarri (2013) menciona que debido al ritmo acelerado de cambios que se operan en nuestra sociedad esto ha afectado a las organizaciones y el desarrollo organizacional ha buscado implementar objetivos y valores fundamentales que la organización debe perseguir:

- Brindar oportunidades para que las personas funciones como seres humanos y no como simples máquinas que operan, así como brindar oportunidades para que cada miembro de la organización y la organización misma desarrollen todo su potencial.

- Procurar la eficiencia del organismo en función de todas sus metas.

- Procurar crear un ambiente en donde se viva un trabajo estimulante y que este ofrezca un reto que vencer.

- Procurar oportunidades a miembros de los organismos que influyan en el desempeño del trabajo en la organización y del medio que lo rodea.

- Tratar a cada ser humano como un conjunto complejo de necesidades los cuales son relevantes para su trabajo y para su vida.

- Respeto por la gente, es decir, que los individuos se consideran responsables, conscientes e interesados y deben ser tratados con dignidad y respeto.

- Confianza y apoyo donde la organización efectiva y saludable se caracteriza por la confianza, la autenticidad, la apertura y el clima de apoyo.

- Búsqueda de la igualdad de poder dejando a un lado el enfatizar la autoridad y el control jerárquicos, por lo que permiten la libertad de ideas y el ejercicio de las mismas.

- Los problemas no deben esconderse debajo de la alfombra, sino deben ser confrontados abiertamente, llamado confrontación.

- Búsqueda inalcanzable de la participación donde las personas afectadas por un cambio participe en las decisiones que lo rodean, más comprometidos estarán en poner en práctica esas decisiones.

\section{Proceso del desarrollo organizacional.}

Hernández, et al. (2011) mencionan que el propósito de la administración del proceso de desarrollo organizacional es garantizar que los esfuerzos de la organización apoyen el proceso, que se conozcan las prioridades de la gerencia y que los esfuerzos sean visibles lo cual se encuentra dirigido al desarrollo de 
nuevos conocimientos sobre la organización y la búsqueda de nuevas formas de enfrentar y resolver los problemas y/o puntos de mejora, así como la creatividad para hacer frente a los cambios que sean necesarios.

El proceso se encuentra conformado por diversas etapas:

1. Diagnóstico organizacional,

2. Desarrollo de planes de acción,

3. Administración del desarrollo organizacional,

El diagnóstico funcional utiliza un proceso de diagnóstico en donde el líder que realizará el estudio obtiene la responsabilidad casi total de los objetivos, métodos y la implementación de los resultados.

Tiene como objetivos:

1. Evaluar la estructura interna formal e informal del sistema de comunicaciones y los diferentes canales de comunicación.

2. Evaluar los sistemas y procesos de comunicación a nivel interpersonal, grupal y departamental.

3. Valorar la eficiencia y la necesidad de la tecnología en la comunicación organizacional.

4. Evaluar el impacto que tienen los procesos de comunicación en la satisfacción en el trabajo, en la productividad, en el compromiso y en el trabajo en equipo, entre otros.

Los resultados de dicho diagnóstico se derivan de diversas técnicas basándose en entrevistas, cuestionarios, análisis de trasmisión de mensajes, análisis de experiencias críticas de comunicación, análisis de redes de comunicación y entrevistas grupales.

El diagnóstico cultural tiene la finalidad de descubrir los principios básicos y los valores de una organización, la forma en la que son compartidos y conocidos por los miembros de la misma y la congruencia que tiene con el comportamiento organizacional.

Realizar un diagnóstico de la organización que revele los aspectos álgidos de las empresas será siempre una preocupación de la gerencia de las mismas, considerando las particularidades que las mismas encierran en cuanto a su forma de gobierno, estructura, formalización, entre otros factores, que las hacen tan especial como importante (Valbuena, Leal y Urdaneta, 2018). 
En la administración del desarrollo organizacional los administradores descubren los hechos que los llevaron a los resultados de las acciones que se realizaron; sin embargo si las acciones no obtuvieron los efectos esperados o bien no se solucionó el problema o se aprovechó la oportunidad es importante que se tomen acciones o también llamadas intervenciones.

\section{Clima Laboral.}

Goncalves (1997) define el clima laboral como el conjunto de percepciones que el trabajador tiene de las estructuras y procesos organizacionales que afectan directa o indirectamente el comportamiento de las personas dentro de una organización. A su vez Likert y Gibson plantearon en 1986 el término clima organizacional para describir la estructura psicológica de las organizaciones. Chiavenato (2000) argumenta que el clima organizacional puede ser definido como las cualidades o propiedades del ambiente laboral que son percibidas o experimentadas por los miembros de la organización y que además tienen influencia directa en los comportamientos de los empleados.

Por su parte Bris (2000) alude que Martín en el año 1999 afirmó que el clima laboral es el resultado de la interacción entre los factores y todos los elementos que integran la organización dentro de un contexto interno y un marco socioeducativo externo.

Brunet (1987) basado en las teorías de los autores antes mencionados considera que el comportamiento en el ámbito laboral corresponde a una respuesta directa de las condiciones organizacionales y también del comportamiento administrativo. Debido a la teoría de Brunet se puede especificar que el clima organizacional afecta a procesos organizacionales y psicológicos e influye en la productividad, en la satisfacción laboral y en el bienestar de los trabajadores.

Weinert (1981) citado en Bris (2000)_ gestionó tres líneas de pensamiento enfocado al clima de las organizaciones:

- El clima sería algo objetivo, tangible, medible de las organizaciones siendo el conjunto de características objetivas de la organización, perdurables y en cierto modo medible que distinguen una entidad de otra.

- El clima desde una visión subjetiva, pero colectiva. El clima viene a ser algo así como la percepción colectiva de la organización en su conjunto y/o de cada uno de sus sectores. Los miembros de las organizaciones comparten una visión global de la institución a la que pertenecen. Clima de trabajo y organizaciones que aprenden. 
- Destaca el sentido subjetivo, pero individual del clima. No es un constructo mental colectivo, sino individual: cada persona elabora su propia visión de la organización y de las cosas que en ella suceden.

Fincher (1983) destaca que el clima organizacional dentro del sistema gerencial o administrativo incluye políticas, procesos, reglas, sistemas de recompensas, toma de decisiones e incluso la estructura organizacional. Asimismo define al clima organizacional como las percepciones que los empleados pueden tener en relación a las prácticas organizacionales destacando que cada organización cuenta con su propia esencia y forma de gestionar la misma.

Realizando una breve síntesis diversos autores explican que el clima laboral se refiere a:

- Las características del medio ambiente de trabajo en que los empleados se desarrollan día a día.

- Sin lugar a duda tiene repercusiones en el comportamiento laboral.

- Por último, es una variable que interviene entre los factores del sistema organizacional y el comportamiento individual.

Las definiciones anteriores nos muestran un panorama amplio de cómo a lo largo del tiempo la sociedad y las organizaciones le han dado un significado al clima laboral. Sin embargo, la definición que se apega más al estudio a realizar fue creada por Méndez (2006) donde destaca que "el clima laboral es el resultado de la forma como las personas establecen procesos de interacción social y donde dichos procesos están influenciados por un sistema de valores, actitudes y creencias, así como también de su ambiente interno". (Solarte, 2009. P.46). Por tal motivo es imperante comprender la estructura de la organización, sus procesos y el comportamiento de las personas.

Bris (2000) en su aportación al estudio del clima laboral nos hace mención que Brunet en el año 1987 hace referencia que el estudio de Likert trae como consecuencia la determinación de diversos tipos de clima laboral:

- Autoritario: clima cerrado, donde existe una estructura rígida por lo que el clima es desfavorable.

- Autoritario explorador: La dirección no posee confianza en sus empleados, el clima que se percibe es de temor, la interacción entre los superiores y subordinados es casi nula y las decisiones son tomadas únicamente por los jefes, 
- Autoritario paternalista: existe confianza entre la dirección y sus subordinados, se utilizan recompensas y castigos como fuentes de motivación para los trabajadores, los supervisores manejan mecanismos de control.

- Participativo: clima abierto con una estructura flexible creando un clima favorable dentro de la organización.

- Consultivo: se caracteriza por la confianza que tienen los superiores en sus subordinados, se les es permitido a los empleados tomar decisiones específicas, se busca satisfacer necesidades de estima, existe interacción entre ambas partes existe la delegación. Definido por el dinamismo y la administración funcional en base a objetivos por alcanzar.

- Participación en grupo: existe la plena confianza en los empleados por parte de la dirección, integración de todos los niveles, la comunicación fluye de forma verticalhorizontal - ascendente - descendente. El punto de motivación es la participación, se trabaja en función de objetivos por rendimiento, las relaciones de trabajo (supervisor supervisado) se basa en la amistad y las responsabilidades son compartidas.

\section{Características y dimensiones del clima laboral.}

En el clima organizacional es evidente el cambio de las actitudes de las personas ya sea de manera definitiva o temporal considerando que pueden ser por diversas razones como son el cierre mensual, los días de pago, los incrementos salariales, los programas de capacitación, reducciones de personal, los cambios organizacionales, etc.

De acuerdo a lo anterior se considera que el clima laboral se caracteriza por:

- El comportamiento de los trabajadores es modificado por el clima de la empresa.

- El clima de la empresa ejerce influencia en el compromiso e identificación de los trabajadores.

- Trabajadores modifican el clima laboral de la organización y también afectan sus propios comportamientos y actitudes.

- Los problemas de rotación y ausentismo pueden ser una alarma para la empresa de que existe un mal clima laboral, es decir, existe insatisfacción. (García e Ibarra, s.f. p. 14) 
Brunet (2004) en su aportación al clima de trabajo en las organizaciones menciona que los efectos del clima laboral pueden resumirse en efectos directos y de interacción. Afirma que los efectos directos se deben a los atributos o propiedades propios de la empresa sobre el comportamiento de los miembros de la misma donde este varía de un clima a otro.

En cambio, los efectos de interacción se refieren a la influencia de los atributos de la organización en personas diferentes, así como también a los apoyos que el ambiente de trabajo le ofrece a cada empleado.

Por lo anterior se dice que el clima laboral tiene una naturaleza multidimensional debido a que las variables que lo componen se interrelacionan y es sumamente difícil poder aislarlas; por lo mismo, los efectos del clima sobre el rendimiento, la satisfacción y la productividad apoyan la naturaleza misma del clima y se suman en consecuencia a las causas de la misma.

Bowers, D.G y Taylor, J.C. (1972) realizarón un instrumento el cual tiene como objetivo medir las características organizacionales apartir de tres variables siendo el liderazgo, el clima organizacional y la satifacción los principaes en función de cinco dimensiones:

1. Apertura a los cambios tecnológicos: basada en la disponibilidad de los individuos a hacerle frente a los nuevos recuros o equipos que la compañía propociona que pueda apoyar a facilitar y mejorar el trbajo de los empleados

2. Recursos humanos: se inclina a la atención que el personal del departamento le brinda a los empleados buscando especialmente el bienestar común

3. Comunicación: son todas aquellas redes de comunicación que interactuan dentro de la organización así como en la factibilidad que se tiene a la apertura tanto de quejas como de sugerencias hacia la organización

4. Motivación: son las condiciones que llevan a los trabajadores a trabajar con mayor o menor entusiasmo en la actividades que desempeñan

5. Toma de decisiones: evalúa a información disponible y utilizada en las decisiones que son tomadas entro de la organización así como el papel que los trabajadores desempeñan dentro del proceso.

Brunet (2004, citado en García, s.f., p. 17) hace mención que los principales cuestionarios que miden la percepción del clima son en función de ocho dimensiones: 
1. Los métodos de mando. La forma en que se utiliza el liderazgo para influir en los empleados.

2. Las características de las fuerzas motivacionales. Los procedimientos que se instrumentan para motivar a los empleados y responder a sus necesidades.

3. Las características de los procesos de comunicación. La naturaleza de los tipos de comunicación en la empresa, así como la manera de ejercerlos.

4. Las características de los procesos de influencia. La importancia de la interacción superior/subordinado para establecer los objetivos de la organización.

5. Las características de los procesos de toma de decisiones. La pertinencia de la información en que se basan las decisiones, así como el reparto de funciones

6. Las características de los procesos de planeación: La forma en que se establece el sistema de fijación de objetivos o directrices.

7. Las características de los procesos de control. El ejercicio y la distribución del control entre las instancias organizacionales

8. Los objetivos de rendimiento y de perfeccionamiento. La planeación, así como la formación deseada.

Brunet (2004) hace mención que diferentes investigadores han abordado de diversas maneras la medida del clima mediante cuestionarios, pero aún no han llegado a conciliar en los tipos de dimensiones más óptimos para evaluar al clima organizacional con el fin de tener la estimación del mismo lo más exacta posible.

\section{Método}

\section{Diseño.}

El objetivo de la investigación es la realización de un diagnóstico organizacional en la empresa de servicios logísticos aplicando la metodología cualitativa a través de los métodos de estudio de caso y la fenomenología.

\section{Instrumentos.}

Atendiendo al paradigma cualitativo y a los métodos de la fenomenología y estudio de caso se utilizaron las técnicas de entrevista semiestructurada, la observación y el análisis de documentos, 
asimismo, se aplicaron encuestas al personal del área administrativa y operativa y se triangularon los resultados con entrevistas al personal directivo.

\section{Procedimiento.}

- La evaluación se aplicó a todo el personal que se encuentra laborando en la actualidad en la oficina de Querétaro (27 personas),

- El análisis nos permite estudiar la percepción que los empleados tienen de su organización, el ambiente laboral y cómo este mismo afecta el desempeño empresarial,

- Entrevista al Branch Manager para la creación del FODA,

- Encuesta evaluando a los 27 integrantes de la oficina de Querétaro de Opinión Individual considerando de una lista de 48 Elementos los 33 más importantes en escala Likert, de acuerdo a la encuesta aplicada por Meza \& Asociados en conjunto con P\&A Consultores de RRHH.

\section{Resultados}

Después de recolectar la información a través de las técnicas de una entrevista conformada por trece preguntas y una encuesta apoyada en un cuestionario de treinta y tres ítems considerando cuatro posibles respuestas en base a la escala Likert donde el encuestado seleccionó la que considero conveniente y se procedió a la interpretación o análisis de cada uno de los valores arrojados. Se procedió a representar de forma gráfica tanto los resultados de la entrevista por medio de un análisis FODA y computarizada el análisis porcentual de las respuestas obtenidas para así proceder a realizar una breve interpretación y análisis de dichos resultados.

\section{Resultados entrevista.}

La entrevista se realizó al Branch Manager de la oficina de Querétaro en el horario laboral con la finalidad de obtener resultados tanto positivos como negativos y también para así poder contar un mayor panorama de las áreas de oportunidad que tiene la compañía y sus miembros. La entrevista fue realizada previamente al otro instrumento de análisis con el objetivo de poder realizar un análisis FODA, poder contar con un panorama general de la situación vivida y también observar las percepciones que tiene el líder sobre sus colaboradores. 
La entrevista realizada brindó la oportunidad no sólo de escuchar la opinión del Branch Manager sino también el poder observar sus reacciones y comportamiento al momento de responder y dar su punto de vista ante las situaciones planteadas.

En cuanto a las fortalezas mencionadas generó un gran énfasis en el bajo nivel de rotación de personal lo cual lo relaciona a que los empleados cuentan con incentivos sumamente atractivos y que la red de subsidiarias a nivel mundial son un gran apoyo para los empleados al momento de realizar sus funciones y que incluso esto mismo impulsa al tener un magnífico trabajo en equipo.

En cuanto a las oportunidades externó que el rubro industrial en Querétaro se encuentra en crecimiento lo cual les beneficia para así poder acrecentar su cartera de clientes y por ende considera que es necesario incrementar las capacitaciones brindadas a los empleados para que estos mismos se encuentren preparados ante las demandas del mercado.

A lo que respecta a las debilidades hizo énfasis en la falta de bases sólidas dentro de la organización como fue el caso de las descripciones de puestos faltantes, en la baja experiencia en la evaluación de desempeño e incluso que el crecimiento tan exponencial que ha tenido en el personal considera que ha influido en la creación de un entorno tenso.

Por último, las amenazas que la organización tiene a la vuelta de la esquina es la fuga de talentos por mayores prestaciones o simplemente porque el trabajo es sumamente demandante y en ocasiones merma la vida personal de los empleados.

\section{Resultados cuestionarios.}

Se envió el cuestionario vía Google Forms a los 27 integrantes de la oficina pertenecientes a los departamentos de Gerencia, Operaciones, Cuentas por Cobrar, Ventas y Servicio al Cliente de las cuales se recibieron en 5 días las respuestas de 26 personas, al ser esta encuesta de carácter confidencial no se tienen datos precisos de las respuestas ingresadas por cada integrante.

El proceso para la ponderación del cuestionario se determinó por medio de 4 respuestas en escala Likert. 
Adaptabilidad: Habilidad para aceptar trabajo bajo diferentes circunstancias, ciudades y tiempos, el $69.2 \%$ de los empleados tienen la total disponibilidad de adaptarse y poder trabajar en una ciudad distinta a donde habitan, a distintas circunstancias que el empleo les exija o bien la adaptabilidad de trabajar en distintos esquemas de tiempo y horario.

Beneficios: Concepto sobre bonos de productividad y ganancias especiales por proyecto. Respecto a la percepción sobre los beneficios que les otorga la empresa, se puede observar que el $88.8 \%$ de los empleados consideran que siempre o frecuentemente los beneficios otorgados como los bonos por productividad son adecuados lo que nos indican que en su mayoría los empleados se encuentran satisfechos con los beneficios económicos que la empresa les brinda.

Desarrollo del empleado: Posibilidad de aprendizaje y desarrollo personal a nivel técnico; Rrespecto al desarrollo que tienen los empleados dentro de la organización, los resultados indican la posibilidad de aprendizaje y desarrollo que los empleados consideran tener dentro de la organización donde el $77 \%$ de los empleados consideran que si se vive un desarrollo profesional en la empresa sin embargo es importante seguir monitoreando que el $33 \%$ restante considera este rubro como negativo.

Cambio: Flexibilidad de la empresa para aceptar cambios y movimientos de personal, se puede observar que más del $60 \%$ de los empleados comparten la misma opinión de que la empresa si muestra flexibilidad para aceptar cambios y movimientos del personal, sin embargo, el 38.5\% de los empleados se sienten atados a la poca probabilidad de poder desempeñar otro puesto de trabajo dentro de la organización.

Compromiso: Compromiso con proyectos y con la empresa en general, las opiniones de los empleados arrojaron que el $\mathbf{8 0 \%}$ de los mismos consideran que existe un compromiso de todos los miembros hacia los proyectos y hacia la organización lo cual es un punto favorable para la organización debido a que es un punto característico de un clima laboral óptimo.

Comunicación: Comunicación por parte de los líderes y dirección en relación al trabajo. Se puede visualizar en el rubro anterior que más del $70 \%$ de la organización considera que existe una buena y efectiva comunicación de los líderes y directores hacia los demás miembros de la organización; sin embargo, es importante tomar a consideración que el $23 \%$ de los miembros opina lo contrario siendo la comunicación uno de los objetivos fundamentales dentro del diagnóstico organizacional funcional. 
Atención al cliente: La empresa busca la satisfacción del cliente en cuanto a resultados. Al evaluar a los empleados con respecto al enfoque que tiene la organización hacia la satisfacción que le brinda esta misma a los clientes fue sumamente notorio que el $92.3 \%$ de los miembros considera este aspecto como fundamental dentro de sus funciones laborales. Es importante recalcar que dicho rubro se caracterizó por ser uno de los elementos más fuertes dentro de la empresa.

Toma de decisiones: La empresa cuenta con la habilidad para decidir cómo realizar movimientos. El $69.3 \%$ de los encuestados consideran que la organización cuenta con un asertivo proceso en su toma de decisiones, sin embargo, el $34.7 \%$ de la población opina lo contrario. Si bien no es un porcentaje alarmante se sugiere mantener este dato en mente para futuros análisis.

Desarrollo de carrera: La empresa motiva a que los empleados sigan preparándose. Los resultados de la gráfica anterior muestran una disyuntiva, es decir, las opiniones se encuentran divididas donde el $46.2 \%$ considera que la empresa no motiva suficiente a los empleados para que sigan preparándose; sin embargo, el resto de los evaluados consideran que sí existe un desarrollo de carrera dentro de la organización y esto es debido principalmente a las distintas expectativas y necesidades de cada uno de los miembros.

Relaciones laborales: La empresa busca más relación entre los empleados. Respecto a la percepción de los miembros el $65.4 \%$ considera que la empresa si busca la interacción entre los empleados dentro de la organización, es decir, la empresa genera actividades para que los empleados convivan con la finalidad de estrechar lazos; sin embargo, el resto tiene un concepto negativo en cuanto a las relaciones laborales que se viven dentro de las instalaciones lo cual sería necesario analizar las variantes.

Equipo: La empresa busca desarrollar el trabajo en equipo. Se puede observar que $70 \%$ de los miembros considera que la organización fomenta el desarrollo del trabajo en equipo mediante actividades de convivencia y debido a que diversos puestos se relacionan entre sí; sin embargo, poco más del $30 \%$ tiene un concepto negativo de este rubro mismo que es considerado uno de los objetivos primordiales dentro del desarrollo organizacional funcional.

Flexibilidad: La empresa cuenta con elementos flexibles de negociación con los empleados. Analizando el nivel de flexibilidad de la organización se identificó que el 50\% de los encuestados consideran que la empresa carece de elementos flexibles de negociación con los empleados; pero por el 
contrario el otro $50 \%$ de la población tiene una imagen positiva de este rubro creando así una disyuntiva en dicho rubro dado a que las percepciones de los empleados se encuentran sumamente disparadas.

Iniciativa: La empresa permite movimientos por parte de los empleados. El $70 \%$ de la población encuestada considera que la empresa cuenta con la iniciativa necesaria para permitir cambios o movimientos del personal dentro de la organización lo cual habla de que es una organización que tiene una mentalidad abierta y dispuesta a que los empleados encuentren el puesto de trabajo óptimo de acuerdo a sus aspiraciones profesionales, económicas, entre otros, sin embargo, para determinar las razones del $30 \%$ de la población que considera este rubro negativo se tendría que realizar una encuesta focalizada.

Satisfacción en el trabajo: Los empleados se sienten satisfechos con su trabajo. Conforme a las percepciones de los empleados el $84.6 \%$ de ellos se encuentran satisfechos con el trabajo que desempeñan dentro de la organización lo cual nos brinda un estadístico positivo debido a que es considerado un aspecto que Hernández et al. (2011) consideran como un objetivo fundamental del desarrollo organizacional.

Liderazgo: Los líderes se muestran como guías de trabajo y decisiones. El liderazgo actualmente es un rubro que las organizaciones buscan alcanzar sin embargo los empleados dentro de Servicios Logísticos H S.A. de C.V. no tiene una percepción muy positiva del mismo dado a que sólo el $61.6 \%$ de los empleados consideran a sus líderes como guías de trabajo y como corresponsables de sus decisiones. Debido a lo anterior es indispensable considerar al 39\% que opina lo contrario para implementar planes de acción.

Reuniones: La empresa realiza reuniones adecuadas y productivas. El mercado global ha hecho énfasis en la importancia de las sesiones entre grupos de personas para la efectiva resolución de problemas o bien para la generación de ideas. En el caso de estudio se puede observar que sólo el 42.3\% de los empleados consideran que las juntas o reuniones que se realizan en el horario laboral son adecuadas y productivas lo que puede llegar a demerita la efectividad de la organización siendo para Newstrom y David (1993) uno de los aspectos que las siete características que el desarrollo organizacional busca alcanzar.

Evaluación de desempeño: Existe un buen método de evaluación de desempeño. En referencia a la perspectiva que tienen los empleados sobre la evaluación de desempeño se cuantificó que el $84.6 \%$ de 
los empleados considera que existe un buen método de evaluación de desempeño aun cuando el proceso es relativamente nuevo para los miembros dado a que se puso en marcha aproximadamente un año; sin embargo, es preciso no dejar a un lado dicho rubro ya que se encuentra ligado con el plan de carrera de los empleados.

Calidad: La empresa cuenta con la habilidad para proporcionar calidad a sus clientes. El $88.4 \%$ de los empleados encuestados consideran que la empresa cuenta con la habilidad para generar calidad a los clientes siendo este aspecto parte de la administración del cambio que Guízar (2013) puntualiza dentro de los elementos que definen el desarrollo organizacional y a su vez se encuentra ligado con el compromiso de los mismos por brindar un óptimo servicio al cliente.

Premios y reconocimientos: Son adecuados, los premios y reconocimientos con el paso de los años han sido considerados incentivos que atraen el compromiso de los empleados hacia la organización. En este rubro el $70 \%$ de los empleados consideran que los reconocimientos o premios que la empresa brinda a los empleados son adecuados. Por lo que respecta al 30\% restante se tendría que realizar una encuesta focalizada para conocer el tipo de premios e incentivos que lograrían motivar a este porcentaje de la población que considera inadecuados los premios y reconocimientos que la empresa actualmente brinda.

Supervisión: Existe buen proceso de supervisión por parte de la empresa. Al analizar la percepción que tienen los empleados en cuanto a la supervisión que se vive dentro de la organización se observa una disyuntiva en los resultados arrojados debido a que el 50\% de los empleados consideran que no existe un correcto proceso de supervisión el cual es un factor que Jiménez (2011) considera relevante dentro del clima laboral; sin embargo la otra mitad de los empleados considera positivo dicho rubro.

Trabajo en equipo: Se vive el trabajo en equipo. El 61.5\% de los empleados tiene la percepción de que el trabajo en equipo se vive frecuentemente dentro del ambiente laboral ya sea para tareas recurrentes como proyectos esporádicos; sin embargo, no se mostraron totalmente seguros de su respuesta y si contemplamos que el $30.8 \%$ de la población considera dicho rubro como negativo es preciso abordar el tema mejorando la comunicación y generando actividades de compañerismo.

Formación: La empresa otorga áreas de desarrollo para el personal. En cuanto a la formación que la organización otorga a los empleados, tan sólo el $50 \%$ de los mismos consideran que la empresa les otorga áreas donde puedan desarrollarse profesionalmente tanto en su actual puesto de trabajo como en brindarles conocimientos para poder crecer en un puesto distinto; sin embargo, el otro $50 \%$ consideran 
dicho rubro como negativo por lo cual es relevante evaluar dichas aportaciones dado a que influye en gran medida las competencias y habilidades de los miembros.

Rotación de personal: Se observa en la empresa movimiento de personal constante. Es preciso recalcar que tan sólo el $25 \%$ de los empleados consideran que existen diversos movimientos de personal, pero el otro $75 \%$ de la población cuentan con una imagen positiva respecto a la baja rotación de personal lo cual reitera la información del análisis FODA donde este rubro se encuentra catalogado como una fortaleza de la organización.

Programa de bienestar: La empresa ofrece apoyos de bienestar personal y familiar. El programa de bienestar fue el rubro en donde el $100 \%$ de los empleados consideraron que existe un programa de bienestar óptimo que la empresa les ofrece a cada uno de los miembros enfatizando en los eventos a nivel nacional que se realizan anualmente y sobre todo en las prestaciones que le otorga a cada miembro

\section{Comentarios sobre la empresa.}

Al finalizar los 33 reactivos de la encuesta se le brindó la oportunidad a encuestados en poder externar sus opiniones, inquietudes con respecto a las vivencias y/o problemáticas percibidas donde gran porcentaje de ellos externo que la compañía es un buen lugar para trabajar sin embargo consideran que requieren mayores programas de capacitación, de trabajo en equipo, que se delimiten responsabilidades entre los equipos, que exista realmente un plan de desarrollo profesional, entre otros aspectos.

A continuación, se muestran algunos de los comentarios textuales de los entrevistados:

- Apoyo, aprendizaje y grandes oportunidades brinda Servicios Logísticos H S.A. de C.V.

- considero que es importante que se delimiten las responsabilidades de cada equipo de trabajo,

- sugiero que se les reconozca a las personas las buenas prácticas que se tienen en el área de trabajo,

- tener más días de vacaciones superiores a la ley mexicana con sustento de ser una empresa alemana,

- me gustaría que existiera un Plan de Crecimiento Profesional y Capacitación para cada empleado, así como una mayor integración, trabajo en equipo y comunicación enfocándonos a 
solucionar problemas reales para hacer un solo frente común ante cualquier problemática interna,

- Servicios Logísticos H S.A. de C.V. es una gran compañía,

- Servicios Logísticos H S.A. de C.V. es una empresa que le gusta que sus empleados se sientan cómodos con el trabajo que desempeñan, y los reconoce. Por ende, es una buena compañía que te permite el desarrollo profesional y personal,

- sin duda, es una muy buena empresa, en donde se aplican los valores morales en todo momento,

- se necesita más trabajo en equipo y asignar responsabilidades,

- Servicios Logísticos H S.A. de C.V. es una excelente empresa en el sentido de compañía (prestaciones) y el personal trabaja por mantener y mejorar el nivel de productividad,

- me parece una excelente empresa donde se permite el aprendizaje continuo y donde realmente se hace un trabajo en equipo,

- los felicito por su gran crecimiento. Sugiero y recomiendo que el ADN se lleve a cabo como sus siglas lo mencionan, ya que en una familia como tal todos tenemos obligaciones y los mismos derechos, es claro que los padres de familia tienen la jerarquía más alta, por tal motivo y en sucesión deben de predicar con el ejemplo,

- mi percepción desde el principio es que el trabajo no es proporcional,

- la sugerencia es que siempre se dé el $100 \%$ de cada persona, las oportunidades se buscan, no llegan por sí solas,

- mayor capacitación al personal.

- Servicios Logísticos H S.A. de C.V. es una magnifica empresa en la cual tienes opciones de crecimiento y te motiva constantemente a desarrollarte de mejor manera dentro de la empresa,

- se requiere mayor involucramiento de los supervisores con sus equipos,

- como recomendación me gustaría que hubiera más capacitación a todos los niveles de manera que podamos crecer al mismo nivel todos,

- en lo personal Servicios Logísticos H S.A. de C.V. es una muy buena empresa y estoy contenta de pertenecer a ella,

- felicidades, somos una gran empresa,

- es necesario mejorar la comunicación en todas las direcciones. 


\section{Análisis global de los resultados.}

Posterior al análisis de los datos obtenidos es necesario jerarquizar los mismos basados en áreas con buena situación, áreas con mejora necesaria y áreas con necesidad de mejora urgente que estos nos marcan un foco rojo que a partir de estos se debe efectuar un plan de trabajo

\section{Áreas con mejora necesaria.}

- Toma de Decisiones

- Relaciones Laborales

- Equipo

- Iniciativa

- Trabajo en Equipo

\section{Áreas con necesidad de mejora urgente.}

- Evaluación de Desempeño: Existe un buen método de evaluación de desempeño,

- Contenido del Empleo y Diseño: La empresa cuenta con metodologías de trabajo,

- Reuniones: La empresa realiza reuniones adecuadas y productivas,

- Flexibilidad: La empresa cuenta con elementos flexibles de negociación con los empleados,

- Supervisión: Existe buen proceso de supervisión por parte de la empresa,

- Formación: La empresa otorga áreas de desarrollo para el personal,

- Comunicación: Comunicación por parte de los líderes y dirección en relación al trabajo,

- Desarrollo de Carrera: La empresa motiva a que los empleados sigan preparándose,

- Trabajo y Vida: La empresa ofrece apoyo en problemáticas de tipo familiar,

- Cambio: Flexibilidad de la empresa para aceptar cambios y movimientos de personal,

- Liderazgo: Los líderes se muestran como guías de trabajo y decisiones,

- Administración: funciona adecuadamente en sus procesos,

- Del Supervisor: Tenemos y contamos con adecuada supervisión,

- Contratación: Se observa constantemente contratación de personal. 


\section{Discusión}

La reflexión derivada de la pregunta de investigación ¿De qué forma el desarrollo organizacional y el clima laboral interactúan entre sí dentro de una empresa de servicios logísticos? es que a través de un plan de intervención derivado de un diagnóstico de clima organizacional se podrán implementar cambios deseados dentro de una organización basados en tendencias de desarrollo, proyecciones y planificaciones que ayuden al desarrollo y bienestar de la misma. Por lo tanto, el diagnóstico es una etapa necesaria de cualquier intervención en las organizaciones y sirve como instrumento adecuado para el conocimiento de la organización y para la planificación racional de su devenir ha sido crecientemente reconocida por los hombres de empresa de nuestro país y del mundo.

\section{Conclusiones}

\section{Plan de acción.}

Como primera fase para poder efectuar los planes de acción correctivos y preventivos en los rubros antes mencionados se realiza un cronograma de actividades determinando el tipo de intervención a emitir, los objetivos a alcanzar, las actividades para lograr dichos objetivos, las personas involucradas en el proceso y fechas de emisión y/o compromiso Nos basaremos en el modelo de intervención de De Faria y los avances se basarán en el modelo Kaizen.

Como segunda fase se añadirán los rubros de los resultados finales y el porcentaje de cambio observado en los participantes siendo la empresa el mejor juez y posteriormente implementar de nueva cuenta el cuestionario impartido en la fase de diagnóstico para evaluar nuevamente los cambios en el desarrollo organizacional.

Es preciso recalcar que las estrategias para el desarrollo organizacional siempre deben aplicarse mediante un análisis previo mediante la identificación de los problemas y que se tengan bases sólidas para implementar las actividades correctivas. 
En el presente estudio se ha expuesto una visión panorámica de los principales temas del diagnóstico organizacional, así como la implementación de este mismo en la empresa Servicios Logísticos H S.A. de C.V. para así determinar las intervenciones necesarias en el clima laboral actualmente vivido en la organización. Durante el diagnóstico se pudo observar que la empresa tiene diversas áreas de oportunidad urgentes que atender tanto en el ramo administrativo, tecno estructurales como en los procesos humanos por lo que se generaron objetivos y actividades de implementación encaminados a cumplir los objetivos de la organización y las necesidades de la misma.

Por último, es importante mencionar que no es sencillo cambiar el clima de una organización dado a que este requiere de tiempo y de la participación de todos los individuos que conforman la organización donde es importante recalcar que los empleados de Servicios Logísticos H S.A. de C.V. ubicados en la Ciudad de Querétaro cuentan con una excelente actitud de mejora continua y se encuentran encaminados a buscar el bien común. A su vez es de suma importancia el involucramiento de la dirección y que esta misma no tenga miedo de poner en tela de juicio los procesos y actividades que actualmente se han llevado a cabo.

\section{Referencias}

Bordas, M. (2016). Gestión Estratégica del Clima Laboral. Madrid: Universidad Nacional de Educación a Distancia, UNED.

Bowers, D. y Taylor, J. (1972). Survey of organizations. Institute for social research. University of Michigan. Michigan.

Bris, M. (2000). Clima de trabajo y organizaciones que aprenden. Educar, (27) ,103-117. DOI: https://doi.org/10.5565/rev/educar.251.

Brunet, L. (2004). El clima de trabajo en las organizaciones: Definiciones, Diagnóstico y Consecuencias. México: Trillas.

Burke, W. (1994). Organizational Development: A process of learning and changing. EUA: Addison-Wesley Publishing Company. 
Chiavenato, I. (2000). Administración de Recursos Humanos. 5a Ed. Colombia: McGraw Hill.

Díaz- Valbuena, N., Leal-Guerra, M. y Urdaneta-Montiel, A. (2018). ADN organizacional y productividad en las empresas familiares. Desarrollo Gerencial, 10 (1), 105- 122. DOI: https://doi.org/10.17081/dege.10.1.2987

De Faria. (2004). Desarrollo Organizacional. Enfoque Integral. México: Ed. Limusa.

Fincher, C. (1983). The Assessment of Institutional Productivity. The Journal of the Association for Institutional. Research, 19(3), 381-384.

García-Ramírez, M., \& Ibarra-Velásquez, L. (s.f.). Diagnóstico de Clima Organizacional del Departamento de Educación de la Universidad de Guanajuato. Recuperado de http://www.eumed.net/librosgratis/2012a/1158/index.htm

Goncalves, A. P. (1997). Dimensiones del Clima Organizacional. Sociedad Latino Americana para la calidad (SLC). Recuperado de http://www.geocities.ws/janethqr/liderazgo/130.html

Guízar, R. (2013). Desarrollo Organizacional Principios y aplicaciones. México: McGraw-Hill.

Hernández, J., Gallarzo, M., y Espinoza, J. (2011). Desarrollo Organizacional Enfoque latinoamericano. México: Pearson.

Jiménez, W. (2011). 9 factores que repercuten en el clima organizacional y los objetivos empresariales. Recuperado de https://www.gestiopolis.com/9-factores-clima-organizacional-objetivosempresariales

Méndez, C. (2006). Clima Organizacional en Colombia. IMCOC: un método de análisis para su intervención. Bogotá, Colombia: Universidad del Rosario. Recuperado de http://repository.urosario.edu.co/handle/10336/914

Newstrom, J. y David, K. (1993). Siete características del Desarrollo Organizacional. México: Editorial Mc. Graw-Hill. 
Porras, J. y Robertson, P. (1992) Organizational Development: Theory, Practice, Research. In: Dunnette, M.D. and Hough, L.M., Eds., Handbook of Industrial and Organizational Psychology, Consulting Psychologists Press, Palo Alto, 719-822.

Solarte, M. (2009). Clima Organizacional y su Diagnóstico: Una aproximación Conceptual. Cuadernos de administración, 25(42), 43-61. DOI: https://doi.org/10.25100/cdea.v25i42.413

Ulibarri, M. (2013, 09 de mayo). Desarrollo Organizacional. Enfoque, Proceso y Objetivos. Visión Industrial. Recuperado de http://www.visionindustrial.com.mx/industria/calidad/desarrolloorganizacional-enfoque-proceso-y-objetivos 